\title{
Detection of biomass burning smoke in satellite images using texture analysis
}

\author{
Koji Asakuma $^{\mathrm{a}, *}$, Hiroaki Kuze ${ }^{\mathrm{a}}$, Nobuo Takeuchi ${ }^{\mathrm{a}}$, Takashi Yahagi ${ }^{\mathrm{b}}$ \\ ${ }^{a}$ Center for Environmental Remote Sensing, Chiba University, 1-33 Yayoi-cho, Inage-ku, Chiba 263-8522, Japan \\ ${ }^{\mathrm{b}}$ Department of Information and Image Sciences, Faculty of Engineering, Chiba University, 1-33 Yayoi-cho, Inage-ku, Chiba 263-8522, \\ Japan
}

Received 26 May 2001; accepted 3 November 2001

\begin{abstract}
Classification results using texture analysis is presented for forest fire smoke from satellite remote sensing data. Texture analysis is carried out for normalized difference images calculated from visible and thermal infrared images of the Indonesian forest fire in 1997. Smoke regions are identified by assuming threshold values for the resulting texture feature as well as for radiances in the original and difference images. It is found that when the thresholds are chosen appropriately for GMS visible and infrared spin scan radiometer, 94\% pixels exhibit agreement between the classification results using the texture analysis and the supervised Euclidean classification. Agreement is found for $96 \%$ pixels in mutual verification using the VISSR image and a concurrent NOAA advanced very high resolution radiometer image. A correlation coefficient of 0.91 is obtained between the results from the two sensors in the variation of the number of smoke pixels accumulated for 12 days in September 1997. Additionally, it is confirmed that as the threshold value of the texture feature is increased, the variation range of the aerosol optical thickness is also increased. As a whole, this study indicates that texture analysis provides quite reasonable results in the smoke detection when appropriately combined with the spectral information. (C) 2002 Elsevier Science Ltd. All rights reserved.
\end{abstract}

Keywords: Indonesian forest fire; Unsupervised classification; Multi spectrum classification; Aerosol optical thickness; GMS VISSR; NOAA AVHRR

\section{Introduction}

Aerosols play an important role in the radiation budget of the earth. Although forest fires in the tropical region are basically local phenomena, they contribute also to the change of the atmosphere of the regional or sometimes even global scale by generating a large amount of aerosol particles (Charlson et al., 1992; Taylor and Penner, 1994). Satellite observation of forest fires is quite useful, since information on smoke aerosols can be obtained for a wide area nearly instantaneously. In this paper, we describe a method to extract regions

\footnotetext{
*Corresponding author. Fax: +81-43-290-3857.

E-mail address: asakuma@ceres.cr.chiba-u.ac.jp (K. Asakuma).
}

that are characterized by high concentrations of smoke aerosols. Conventional methods such as the multi-level slicing and the most-likelihood method have usually been used to classify pixels in satellite images (Lubin and Morrow, 1998; Nair et al., 1998; Berendes et al., 1999). When the object to be analyzed is distributed dispersively, on the other hand, the use of texture analysis combined with multi-spectral information has been proved to be valuable (Weszka et al., 1976). Texture analysis is capable of exploiting the spatial features more directly and more systematically than other statistical methods such as that based on the coefficient of variation. A variety of quantities describing spatial features have already been derived and discussed in the literature. Clouds have often been analyzed using satellite remote sensing data. For example, Ebert 
(1987) described a pattern recognition technique for distinguishing cloud types in the polar regions, Kuo et al. (1988) applied the method to the detection of high cirrus clouds, and Key (1990) used it to discriminate between clouds and snow/ice regions on the ground.

Christopher et al. (1996) analyzed the Amazon biomass burning by means of texture analysis. They extracted possible burning areas by combining spectral signatures in visible and thermal-infrared images, and subsequently choosing the portions that were characterized by uniform values of reflected radiance (i.e. showing no spatial pattern expected for ground surfaces). This latter extraction was done by texture analysis to remove the pixels exhibiting the reflectance from ground surfaces. The framework of our present work is basically similar to that of Christopher et al. (1996), but with more quantitative examination of the texture analysis applied to smoke detection.

The purpose of the present paper is twofold. First, taking the Indonesian forest fire in 1997 as a case study, we implement automatic smoke extraction on two types of satellite images with different spatial resolutions. The results of classification are compared with each other as well as with that of the visual classification. Second, we examine the aerosol optical thickness for regions that are classified as homogenous in the texture analysis to study the extent to which the aerosol optical thickness is actually uniform. This is accomplished by separately deriving the optical thickness over the sea area from relevant satellite images. Appropriate images of the visible and infrared spin scan radiometer (VISSR) of the GMS-5 satellite and the advanced very high resolution radiometer (AVHRR) of the NOAA-14 satellite are employed for the study of smoke extraction. The latter images are used to calculate the aerosol optical thickness, because of the availability of the sensor calibration.

Satellite data have so far often been used for the analysis of forest fires, such as those in Amazon, Africa, and Indonesia. In these previous investigations, principal interests consisted in the derivation of optical and chemical properties of smoke aerosols (Kaufman and Holben, 1996; Holben et al., 1996; Liousee et al., 1997) and estimation of the radiation forcing on the basis of their optical characteristics (Kaufman et al., 1990; Prins and Menzel, 1994). These objectives are different from the viewpoint of the present paper, i.e. mutual validation of the smoke-extraction results and the study of the relationship between the textural feature value and the aerosol column amount.

This paper is organized as follows. In Section 2.1 smoke areas are extracted by texture analysis of the difference image between the GMS-5 VISSR visible image $(0.5-0.9 \mu \mathrm{m}$ band) and infrared image (10.5$11.5 \mu \mathrm{m}$ band). Also, we prepare a reference image by means of supervised classification using the standard
Euclidean distance. Comparison between the automated (textural) and visual (supervised) results is made in Section 3.1. In Section 2.2 textural analysis is applied to the difference image between the channel 1 (580$680 \mathrm{~nm})$ and channel $4(10.3-11.3 \mu \mathrm{m})$ images of NOAA-14 AVHRR. This result with a high spatial resolution $(1.1 \mathrm{~km})$ is compared with the VISSR case (5 km resolution) in Section 3.2. In Section 2.3 we derive the aerosol optical thickness over the sea area from the AVHRR channel 1 image. This result is discussed in Section 3.3 in relation to the threshold value assigned to the textural mean. In Section 3.4 we discuss the information obtained by combining the textural feature value and the aerosol optical thickness, and in Section 3.5 the utility of the texture feature to detect inhomogeneity in the smoke detection.

\section{Methodology}

\subsection{Smoke detection in GMS VISSR images}

\subsubsection{Non-supervised classification using texture analysis}

We adopt the gray level difference vector (GLDV) in order to make the calculation simpler than the case using the co-occurrence matrix (Weszka et al., 1976; Christopher et al., 1996). The following textural mean is employed to evaluate the homogeneity of pixel radiance in each window $(9 \times 9$ pixels $)$ scanned in an image:

$f_{\mathrm{m}}(r, \theta)=\sum_{n=0}^{N_{\mathrm{g}}-1} n P_{\mathrm{g}}(n)_{r \theta} / N_{\mathrm{g}}$,

where $r$ and $\theta$ denote the distance and directional angle between two pixels, $N_{\mathrm{g}}$ is the division in the radiance level, and $P_{\mathrm{g}}$ is the probability that the difference of the radiance between the two pixels becomes $n$. The textural feature $f_{\mathrm{m}}(r, \theta)\left(0<f_{\mathrm{m}}(r, \theta)<1\right)$ represents the variance of pixel radiance in the window, approaching 0 when the homogeneity is high.

The following parameter, $D_{\mathrm{GMS}}\left(-1 \leqslant D_{\mathrm{GMS}} \leqslant 1\right)$, is calculated for each pixel, and the resulting image is subjected to texture analysis:

$$
D_{\mathrm{GMS}}=\left(4 u_{\mathrm{g}}-v_{\mathrm{g}}\right) /\left(4 u_{\mathrm{g}}+v_{\mathrm{g}}\right),
$$

where $u_{\mathrm{g}}$ is the digital number (DN) of the visible channel, and $v_{\mathrm{g}}$ that of the infrared channel. The DN of the visible channel is multiplied by a factor of 4 so that its variation range matches with that of the infrared channel. We define a texture-analyzed image (TAI) as what we obtain by applying Eq. (1) to this difference image. We performed the actual data processing by transforming the TAI into an 8-bit image: for simplicity, however, we employ the numerical value that is directly calculated by Eq. (2) in the following analysis. 
Regions with radiance contributions from fire smoke are detected in the following manner. First we make use of the spectral feature that fire smoke would exhibit. Since its temperature is relatively high and it shows high reflectivity in the central part of the visible wavelength, the value of $D_{\mathrm{GMS}}$ in Eq. (2) is considered to be large (Christopher et al., 1996; Ackerman and Chang, 1992; Rao et al., 1997). Furthermore, setting a lower limit to the temperature detected by the infrared sensor attains reasonable discrimination of the smoke against clouds. Then, the smoke region is characterized by the uniformity of the textural feature in Eq. (1) in the window. Combined together, these three conditions are expressed as

$$
\begin{aligned}
& D_{\mathrm{GMS}}>0.2, \\
& v_{\mathrm{g}}<145, \\
& f_{\mathrm{m}}(r, \theta)<\delta_{\mathrm{GMS}} .
\end{aligned}
$$

In the first condition, the threshold value $(0.2)$ is empirically determined to distinguish between the smoke and background (land and sea) surfaces (see also Fig. 6 below). In the second condition, it should be noted that a smaller value of $v_{\mathrm{g}}$ indicates a higher brightness temperature $\left(v_{\mathrm{g}}<145\right.$ means that brightness temperature is higher than $280 \mathrm{~K}$ ). In the third, the threshold value $\delta_{\mathrm{GMS}}$ is changed to examine how it affects the smoke retrieval. We define a smoke-extracted image (SEI) by what we obtain by applying these conditions to the TAI and transforming it into a binary image. The resulting image will be shown and discussed later in Section 3.1.1.

\subsubsection{Supervised classification with standard Euclidean distance}

In this subsection, we describe the method to visually classify the GMS image by means of the supervised classification. We define the classes as listed in Table 1, and each training area is sampled as exemplified in Fig. 1. Originally this figure is a false color composite obtained by assigning red and green to the GMS visible channel and blue to its thermal infrared channel: as a result, the sea, vegetation, cloud, and smoke areas become blue, green, white, and yellow, respectively. In particular, the smoke areas (AG and AO) were sampled on Kalimantan and surrounding sea areas. The vegeta-

Table 1

Training classes

\begin{tabular}{llll}
\hline Index & Class name & Index & Class name \\
\hline AG & Smoke over the ground & OC & Ocean \\
AO & Smoke over the ocean & VE & Vegetation \\
CT & Thin cloud & DS & Desert \\
CG & Cloud over the ground & SG & Sun glitter \\
CO & Cloud over the ocean & & \\
\hline
\end{tabular}

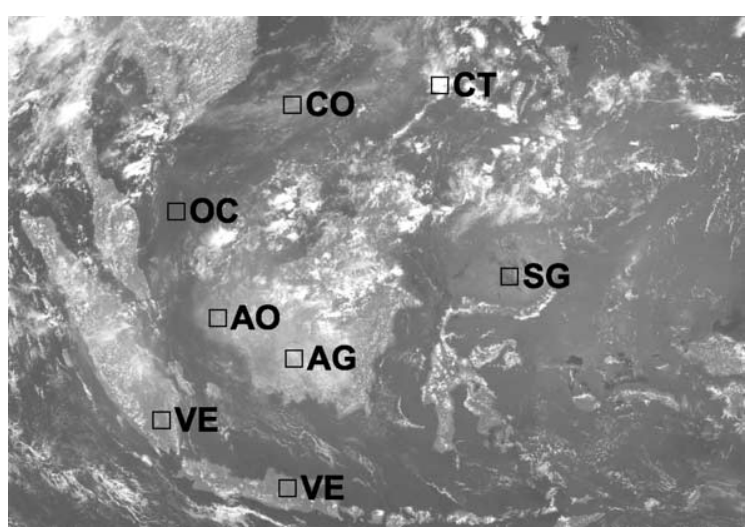

Fig. 1. A set of training areas sampled for the classes in Table 1 (image size is $1200 \times 800$ pixels) (2 September 1997).

tion areas were determined with the help of geographical information. We analyzed an image per day in September 1997; the local time was fixed at 11:00 in agreement with the overpass of the NOAA satellite. A total of 30 images were analyzed, and for each class $k$ in Table 1, about 20 regions were sampled in each image. By using the visible and infrared radiance values of $u$ and $v$, monthly values of the average $U_{k}=\left(\bar{u}_{k}, \bar{v}_{k}\right)$, standard variation $S_{k}=\left(\sigma_{u k}, \sigma_{v k}\right)$, and the correlation between the two $\left(C_{u v}\right)$ were calculated. Since it turned out that the correlation was insignificant $\left(C_{u v}<0.056\right)$, we employed the standard Euclidean distance rather than the Mahalanobis' generalized distance, which is suitable for the analysis of correlated cases. The result will be explained in Section 3.1.2.

\subsection{Smoke detection in NOAA AVHRR images}

The NOAA AVHRR images have higher spatial resolution than the GMS VISSR images. As before, texture analysis is implemented for the normalized difference image calculated from the visible (channel 1; $0.58-0.68 \mu \mathrm{m}$ ) and thermal infrared (channel $4 ; 10.3-$ $11.3 \mu \mathrm{m})$ channels:

$D_{\mathrm{NOAA}}=\left(u_{\mathrm{n}}-v_{\mathrm{n}}\right) /\left(u_{\mathrm{n}}+v_{\mathrm{n}}\right)$.

Here, $u_{\mathrm{n}}$ and $v_{\mathrm{n}}$ stand for the DN of channel 1 and 4, respectively (no enhancement factor is introduced in this case). Note that the width of this visible channel is much narrower than the VISSR visible channel $(0.5-0.9 \mu \mathrm{m})$. The spatial resolution of the thermal infrared channel $(1.1 \mathrm{~km})$ is 4.5 times better than that of the VISSR thermal infrared channel. Thus one can expect increase in the accuracy of smoke detection for AVHRR, though the observation is more infrequent for AVHRR than for VISSR. 
The following three conditions are applied to extract the smoke area:

$D_{\mathrm{NOAA}}>0.2$,

$v_{\mathrm{n}}<200$,

$f_{\mathrm{m}}(r, \theta)<\delta_{\mathrm{NOAA}}$.

The second condition means that the brightness temperature in the thermal infrared band is higher than $280 \mathrm{~K}$, as in the case of VISSR. The result will be given in Section 3.2.

\subsection{Derivation of aerosol optical thickness from AVHRR images}

The satellite image can also be exploited to derive the aerosol optical thickness, which is an essential parameter to check the uniformity of the smoke column amount. Here we use the dark target method to estimate the optical thickness (Nakajima and Higurashi, 1997; Ru et al., 2000; Asakuma et al., 2000). Radiation components are calculated by the algorithm and aerosol models incorporated into the $6 \mathrm{~S}$ code (Vermote et al., 1997a,b), a versatile code developed for the purpose of atmospheric correction of satellite data. Sea areas in the NOAA AVHRR channel 1 images are analyzed to obtain the smoke optical thickness at $550 \mathrm{~nm}, \tau_{550}$.

In the $6 \mathrm{~S}$ code, the apparent reflectance $\rho^{*}$ that corresponds to the radiance observed by a satellite sensor is expressed by using the reflectance from the atmosphere $\rho_{\mathrm{a}}$, the reflectance from the vicinity of the target $\rho_{\mathrm{e}}$, and the target reflectance $\rho_{\mathrm{c}}$ as

$\rho^{*}=\rho_{\mathrm{a}}+\frac{\mathrm{e}^{-\tau / \mu_{\mathrm{s}}}+t_{\mathrm{d}} \downarrow}{1-\rho_{\mathrm{e}} S}\left\{\rho_{\mathrm{c}} \mathrm{e}^{-\tau / \mu_{\mathrm{v}}}+\rho_{\mathrm{e}} t_{\mathrm{d}} \uparrow\right\}$.

Here $\theta_{\mathrm{s}}$ and $\theta_{\mathrm{v}}$ are the solar and satellite zenith angles, respectively, $\mu_{\mathrm{s}}=\cos \theta_{\mathrm{s}}$ and $\mu_{\mathrm{v}}=\cos \theta_{\mathrm{v}}$ are their cosines, $t_{\mathrm{d}} \downarrow$ and $t_{\mathrm{d}} \uparrow$ are the downward and upward diffuse transmittance, respectively, $S$ the spherical albedo of the atmosphere, and $\tau$ denotes the optical thickness of the atmosphere at the observation wavelength. If we give $\theta_{\mathrm{s}}, \theta_{\mathrm{v}}, \phi$ (the relative azimuth between the satellite and solar directions), $\rho_{\mathrm{c}}, \rho_{\mathrm{e}}$, and $\tau_{550}$ as input parameters to the $6 \mathrm{~S}$ code, and select an appropriate standard molecular profile and an aerosol model, the code calculates $\rho_{\mathrm{a}}, t_{\mathrm{d}} \uparrow, t_{\mathrm{d}} \downarrow$, and $S$. Subsequently, Eq. (6) gives us the reflectance at the top of the atmosphere. In the case of sea areas treated here, we assume the reflectance of the target and adjacent pixels to be the same $\left(\rho_{\mathrm{e}}=\rho_{\mathrm{c}}\right)$ : this value is assumed to be $2 \%$ for AVHRR channel 1 so that we obtain a result in agreement with the ground observation (see Section 3.3).

In Fig. 2, retrieval points are designated as a-d near Kalimantan. The results at these points will be used in Section 3.4 to discuss the relationship between the textural mean and the uniformity of the aerosol column amount. Similarly, the variation of $\tau_{550}$ is also derived at point A above sea near Singapore. The monthly change of $\tau_{550}$ at this point during November 1997 is used for the validation of the present method against the sky radiometer data obtained at Singapore.

Fig. 3 shows an example of the relationship between $\rho^{*}$ and $\tau_{550}$. This is a result computed from the data at point A in Fig. 2 (10 November 1997) with $\theta_{\mathrm{s}}=48.4^{\circ}$, $\theta_{\mathrm{v}}=0.5^{\circ}$ and $\phi=26.9^{\circ}$. We adopted tropical model for the atmospheric profile and forest-fire smoke model for the aerosol model in the $6 \mathrm{~S}$ calculation. The aerosol optical thickness $\tau_{550}$ is estimated from this figure by equating the observed apparent reflectance

$\rho_{1}^{\mathrm{NOAA}}=S_{1} u_{1}+I_{1}$

to $\rho^{*}$. Here, $S_{1}$ and $I_{1}$ are, respectively, the slope and intercept calibration constants of the AVHRR channel 1 (Rao and Chen, 1996). Similar procedures are applied to derive the aerosol column amount from the satellite data of the Indonesian forest fire. The results will be presented and discussed in Section 3.3.

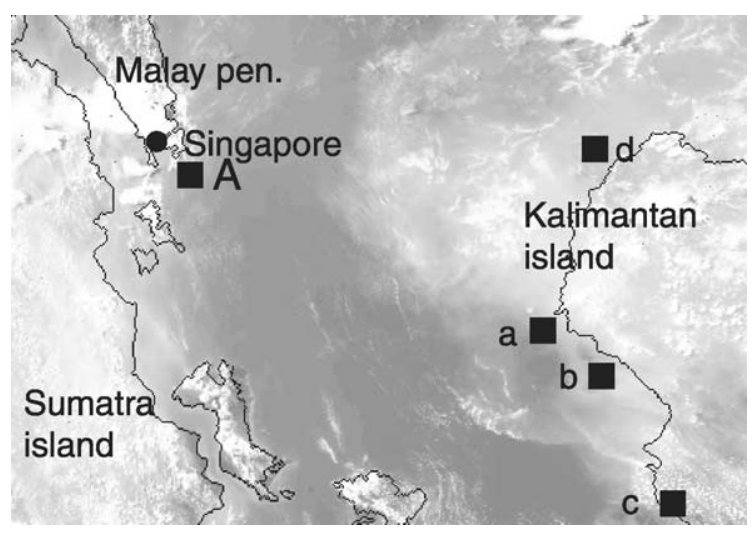

Fig. 2. AVHRR ch. 1 image on 10 November 1997. The monthly change of $\tau_{550}$ (aerosol optical thickness at $550 \mathrm{~nm}$ ) at point A is compared with the result of Singapore skyradiometer measurement. Points a-d, located in the sea area near Kalimantan, are the retrieval points of the aerosol optical thickness to study the aerosol homogeneity.

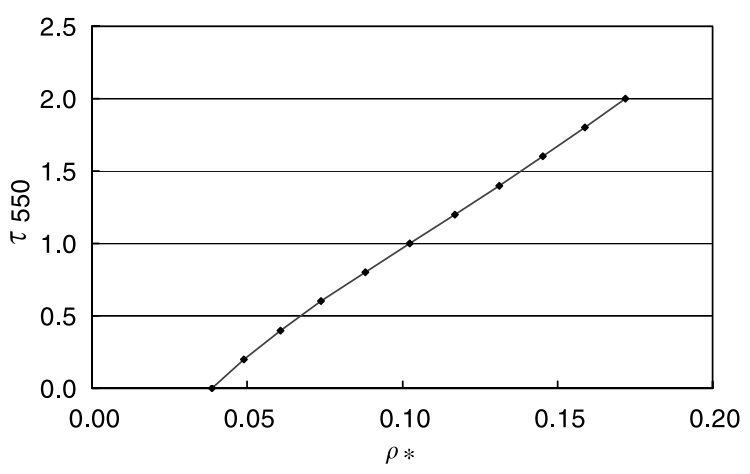

Fig. 3. Relationship between $\tau_{550}$ and $\rho^{*}$ at point A in Fig. 2 (10 November 1997). 


\section{Validation of the smoke extraction and discussion}

\subsection{GMS images}

\subsubsection{Texture analysis}

The difference image on 2 September 1997 between the visible and thermal infrared channels of GMS VISSR is shown in Fig. 4. Note that the spatial resolution of Fig. 4(a) (conformed to the visible resolution) is four times better than that of Fig. 4(b) (conformed to the thermal infrared resolution). The fire smoke is recognized as the white area located at the south part of Kalimantan and extending toward the northwest direction. Texture analysis is applied to Fig. 4(b), and the result is shown in Fig. 5. In this case the GLDV vectors are calculated with $r=1, \theta=$ $0^{\circ}, N_{x}=N_{y}=9$ and $N_{\mathrm{g}}=256$ (8 bit). The condition of $r=1$ is employed to make comparisons among contiguous pixels. In addition to $\theta=0^{\circ}$, we also tested the angular parameter of $\theta=90^{\circ}$ and $\theta=45^{\circ}$ (for which the distance $r$ is increased by a factor of $\sqrt{2}$ ), but no recognizable effects were detected. Therefore, as far as this case is concerned, spreading of smoke is diffusive, with little influence from the wind field. Under such situations, textural mean in Eq. (1) works well as a discriminator of the fire smoke against background land surfaces. The present texture size of $N_{x}=N_{y}=$ $9(40 \mathrm{~km} \times 40 \mathrm{~km})$ is chosen in consideration of the spreading of smoke from a single fire spot: a larger size would result in involvement of unnecessary information, while a smaller size may suffer from lack of statistics.

In Fig. 5, black regions are considered to represent the homogenous regions in the difference image. Fig. 5(a) is the result of the simple texture analysis. It is confirmed in this figure that although the smoke regions are extracted, we need an additional measure to distinguish the smoke from the sea surface, which also displays a homogenous feature in this image. Application of conditions in Eq. (3) removes the sea surface areas and extracts the smoke areas, as indicated in Fig. 5(b) $\left(\delta_{\mathrm{GMS}}=0.3\right)$. The effect of the threshold value, $\delta_{\mathrm{GMS}}$, is discussed in the next subsection.

\subsubsection{Comparison of smoke extraction results by Euclidean classification and texture analysis}

In Fig. 6, we show a map in the DN space clustered with the method described in Section 2.1.2. This map is for GMS images around Indonesia as illustrated in Fig. 1. Fig. 6 is obtained by means of the standard Euclidean distance. For the sake of comparison, the region that satisfies the first and second conditions in Eq. (3) $\left(D_{\mathrm{GMS}}>0.2\right.$ and $\left.v_{\mathrm{g}}<145\right)$ is also illustrated (below the horizontal line and right side of the tilted line). In this region of the map, sun glitter (SG) and desert (DS) become relevant only for more expanded area scenes. The designated region covers most part of the categories of $\mathrm{AO}$ and $\mathrm{AG}$, smoke over the ocean and smoke over the ground. The reason that part of the AO region is found left side of the tilted line is primarily due to contamination from the ocean (OC). This is unavoidable in the visual determination of the training areas, though only a limited number of pixels are actually classified into this "false" category (see below). Another important aspect to be noted here is the presence of vegetation (VE) and a small portion of cloud over the ocean $(\mathrm{CO})$ in the region specified by the quoted conditions. In order to detect smoke regions efficiently, these features should be distinguishable by means of texture analysis.

An example of the present supervised classification is illustrated in Fig. 7(a). The method is applied to the area on and around Kalimantan (2 September 1997). Gray regions depict the fire smoke in this binary image. Black pixels are those belonging to AO but being classified in the "false" region of $D_{\mathrm{GMS}}<0.2$ : they represent only $5.8 \%$ of the entire smoke region $(\mathrm{AO}+\mathrm{AG})$. In
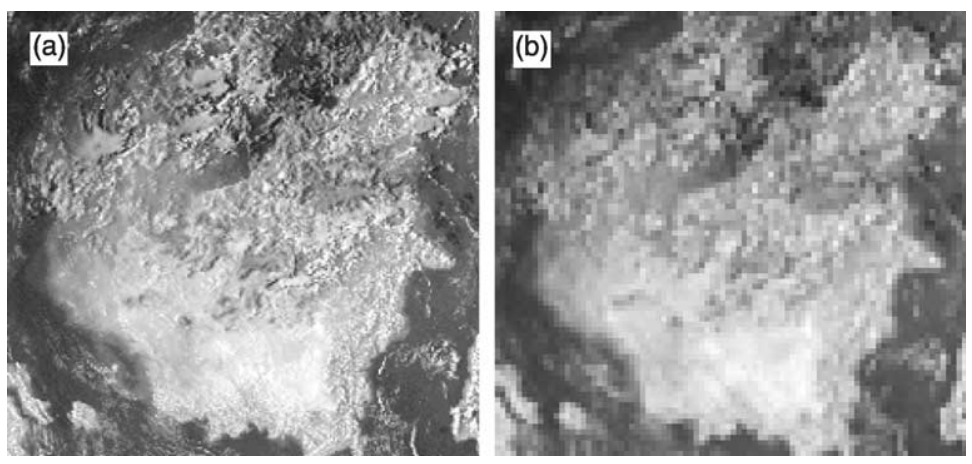

Fig. 4. Normalized difference images obtained with GMS-5 visible and thermal infrared channels (2 September 1997). Image size is conformed to (a) that of the visible image $(350 \times 335$ pixels $)$ and (b) that of the thermal infrared image $(88 \times 83$ pixels $)$. 


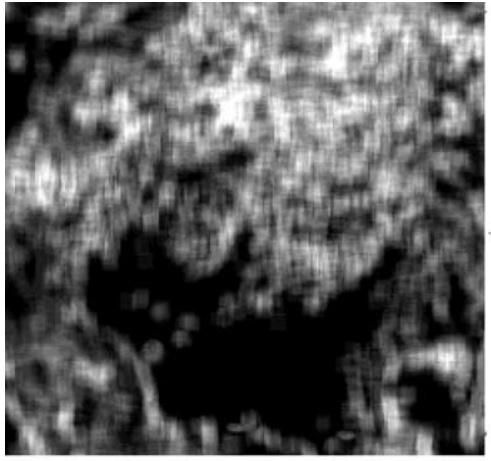

(a)

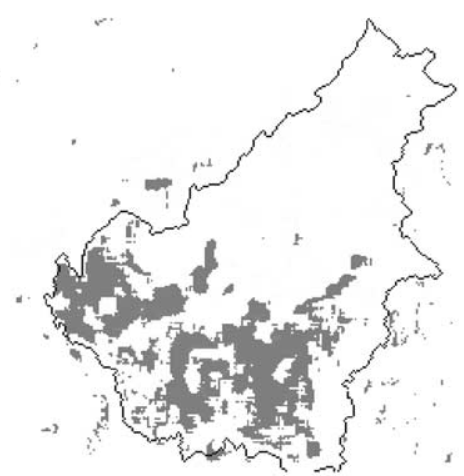

(b)

Fig. 5. (a) Texture-analyzed image (TAI) calculated from the difference image shown in Fig. 4 (2 September 1997). Image size is $88 \times 83$ pixels. (b) Smoke-extracted image (SEI) obtained with $\delta_{\mathrm{GMS}}=0.3$.

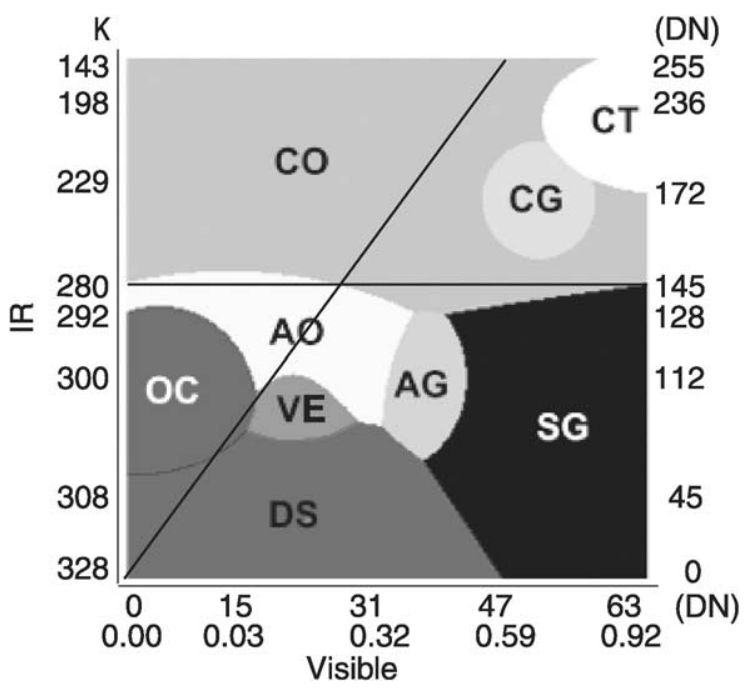

Fig. 6. Cluster map from GMS-5 images in September, 1997, obtained with the standard Euclidean distance. The lines define a region that satisfies the first and second conditions in Eq. (3) (see text).

Fig. 7(b)-(f) we show the binary images (SEI) obtained with various values of the threshold parameter $\delta_{\mathrm{GMS}}$ in Eq. (3). When $\delta_{\mathrm{GMS}}=0.2$ (Fig. 7(b)), only a very limited portion of the image is categorized into the smoke area. When $\delta_{\mathrm{GMS}}=0.4$ (Fig. 4(f)), on the other hand, smoke areas overlap with each other, with no clear separation between the neighboring areas. The SEI with $\delta_{\mathrm{GMS}}=0.3$ (Fig. 7(d)) reasonably agrees with Fig. 7(a) regarding the smoke distribution. In fact when all the pixels are considered on the Kalimantan island, agreement in the classification of pixels turns out to be $94 \%$ between Fig. 7(a) and (d). In the northern part of Kalimantan, more pixels are categorized into smoke pixels in Fig. 7(a)
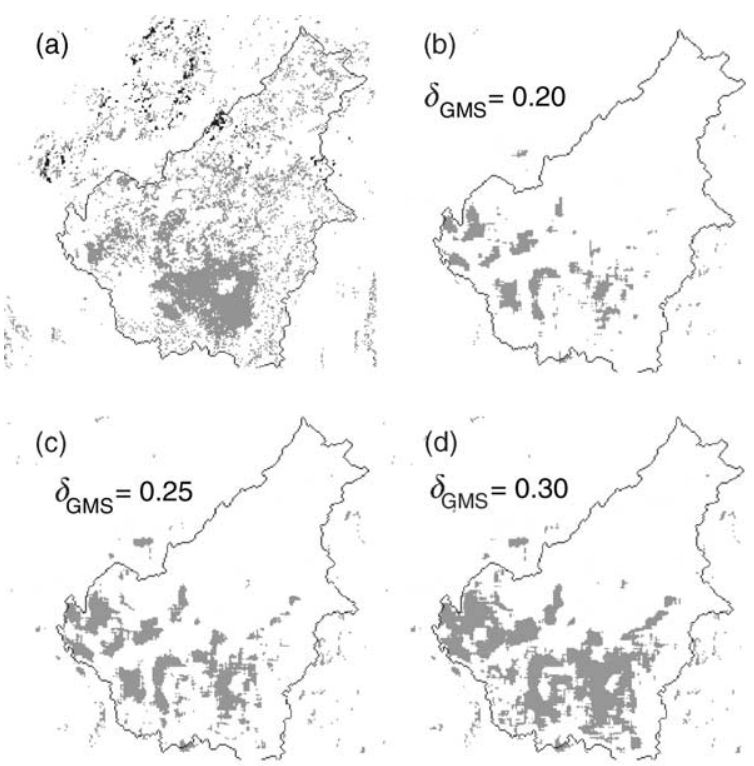

(e)

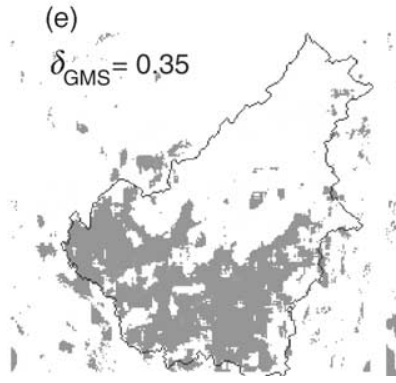

(f)

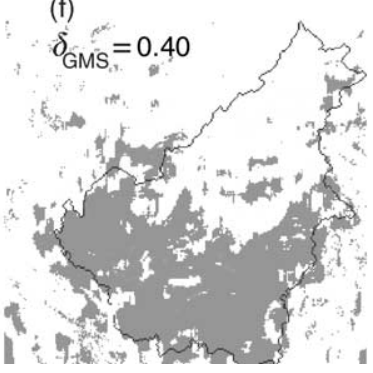

Fig. 7. Smoke-extracted images (SEI) (2 September 1997). (a) Result by the standard Euclidean classification, and (b)-(f) by textural analysis for various values of $\delta_{\mathrm{GMS}}$.

than in Fig. 7(d). This is ascribed to the fact that in texture analysis structures smaller than the window size $(9 \times 9)$ tend to be excluded because of the threshold condition of $f_{\mathrm{m}}(r, \theta)<\delta_{\mathrm{GMS}}$. 

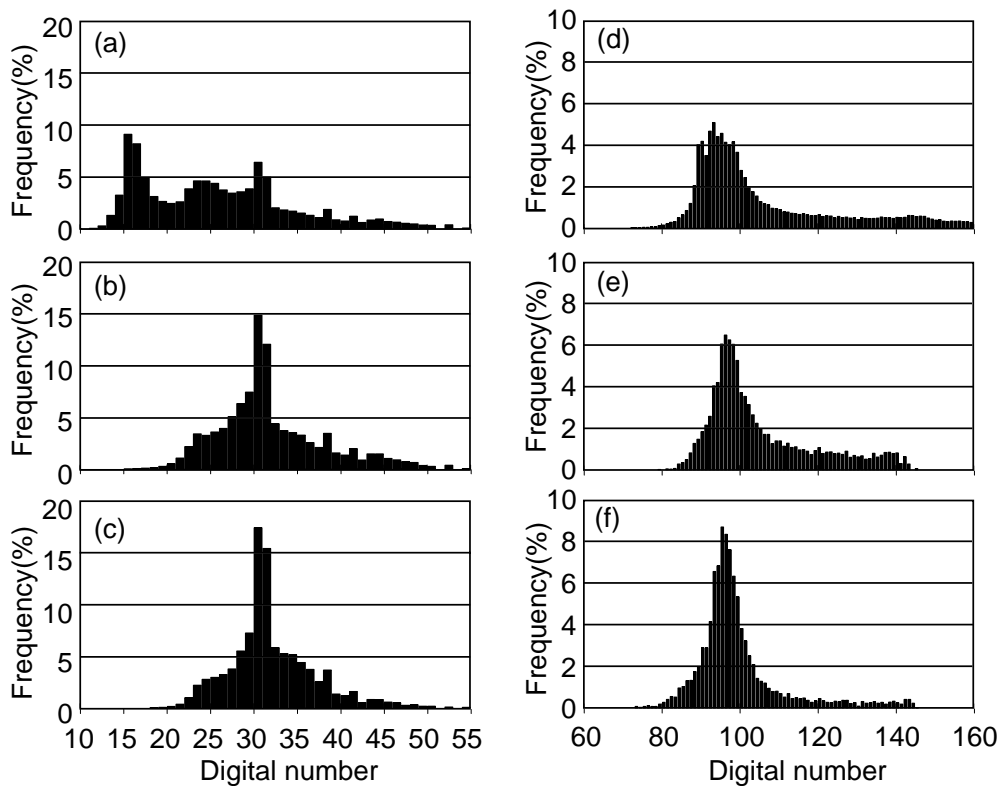

Fig. 8. (a)-(c) Histograms from the GMS visible channel, and (d)-(f) those from the thermal infrared channel. (a) and (d) are for all pixels in September 1997, (b) and (e) results by the standard Euclidean classification, and (c) and (f) those by the texture analysis.

\subsubsection{Validation of classification results using histograms}

Histograms are generated for the visible and thermal infrared channels of GMS VISSR on the basis of the $\mathrm{DN}$ of pixels in the images classified using the supervised classification and the texture analysis. In Figs. 8(a)-(c) and (d)-(f), we show the results for the visible and thermal infrared channels, respectively: (a) and (d) illustrate distributions obtained from all the images in September 1997. In Fig. 8(b) and (e) are for the areas that have been classified as the smoke region in the supervised classification, while (c) and (f) correspond to the smoke region in the texture analysis with $\delta_{\mathrm{GMS}}=$ 0.3. Comparison between (b) and (c) (visible band) indicates that the supervised and texture results are in good agreement regarding the mode value of the DN. The standard deviation for the former is 13.76 , whereas it is 11.56 for the latter. Similarly, the standard deviation of (e) (supervised) is 20.23, while that of (f) (texture) is 16.84. Thus, for both the visible and thermal infrared bands, histograms from texture analysis is found to be more centered around the mode than those from the supervised classification.

In texture analysis, the width of each histogram varies in accordance with the threshold $\delta$. Nevertheless once the GLDV vectors are computed, it is easy to study the effect of this parameter on the classification result. In the supervised classification, on the contrary, determination of the training class is more or less made on a subjective basis, and it is laborious to examine a large number of sample data.

\subsection{Comparison of $A V H R R$ and VISSR results of texture analysis}

Fig. 9(a) displays the normalized difference image between the channel 1 and channel 4 of AVHRR on 2 September 1997. Compared with the VISSR case shown in Fig. 4, smoke fire (white pixels) extends more toward the sea area northwest of Kalimantan. This is presumably due to the difference in the wavelength bands in the two sensors (see Section 2.2), since it is unlikely that the difference in the spatial resolution influences the smoke distribution in this way.

In Fig. 9(b), we show the TAI obtained from the difference image of Fig. 9(a), with $r=1, \theta=0^{\circ}, N_{x}=$ $N_{y}=9$ and $N_{\mathrm{g}}=256$. Compared with the VISSR result in Fig. 5, areas with uniform reflectance (black regions in the binary image) are more separately distributed over Kalimantan. This is presumably ascribed to the better spatial resolution of AVHRR. In addition, it is seen that a larger number of smoke areas appear over the sea area, particularly in the northwest area of the island. This reflects the difference in the smoke distribution over the sea area found in the difference images, Fig. 4 for VISSR and Fig. 9(a) for AVHRR, as mentioned above. 

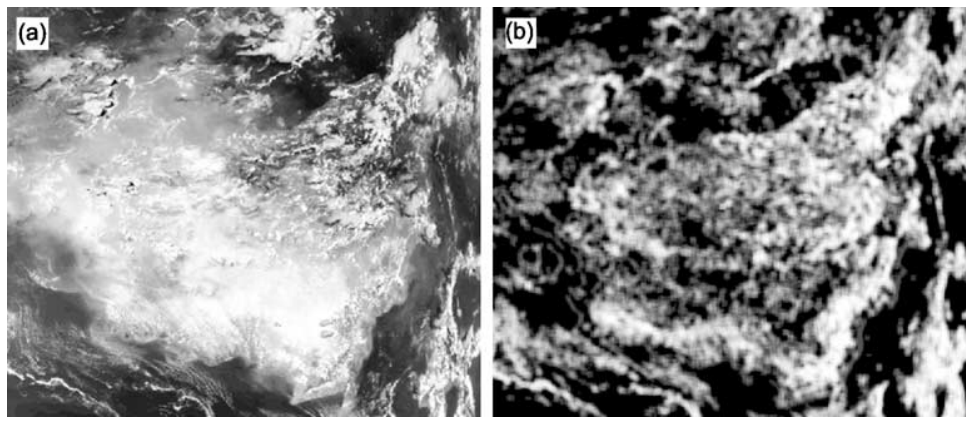

Fig. 9. (a) Normalized difference image obtained from AVHRR ch. 1 and ch. 4 (2 September 1997). Image size is $376 \times 326$ pixels, extracted from the original size of $1024 \times 1024$ pixels. Texture analysis is conducted on the original image. (b) TAI from the difference image in (a).
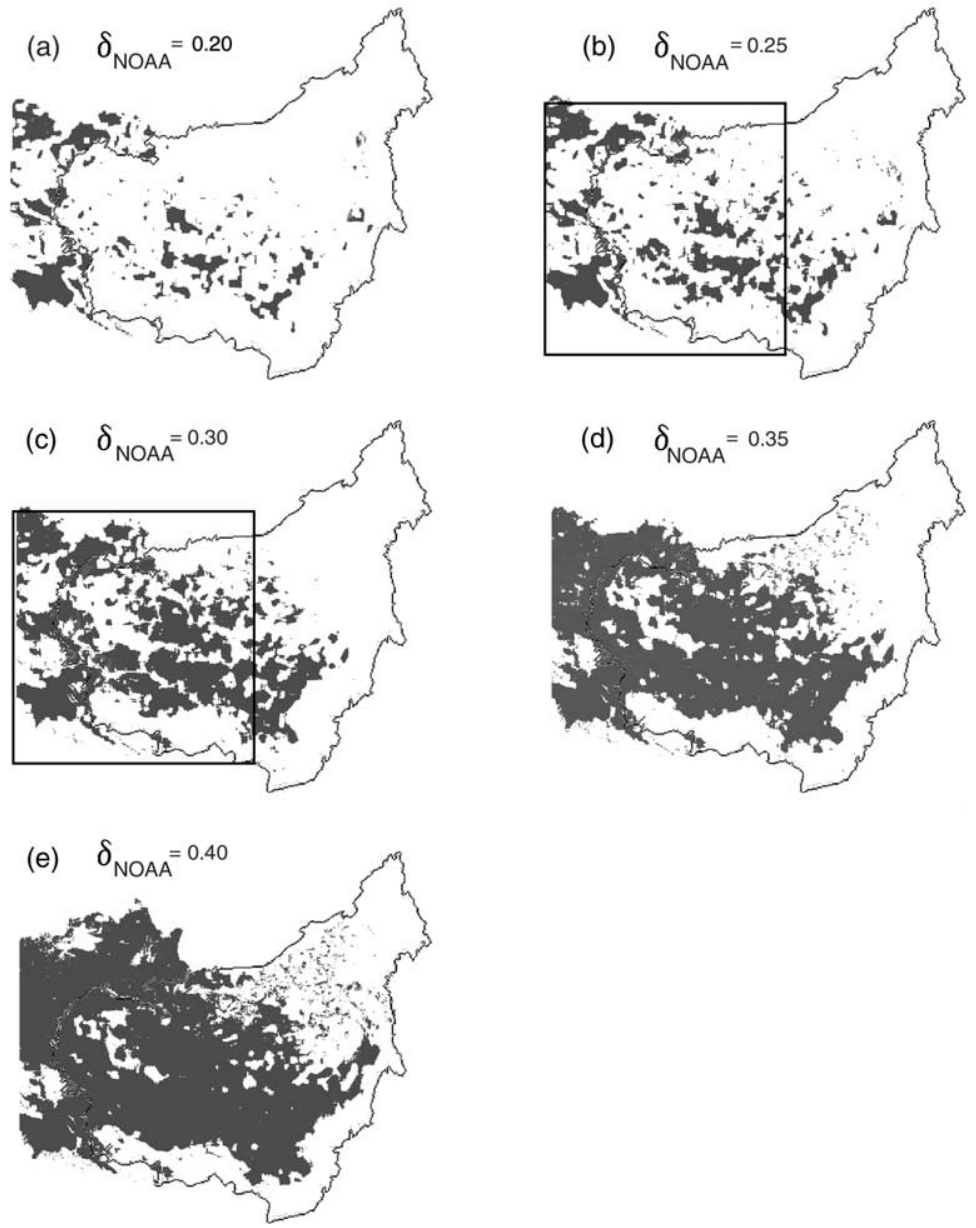

Fig. 10. SEI obtained by using Eq. (5), with (a) $\delta_{\mathrm{NOAA}}=0.2$, (b) $\delta_{\mathrm{NOAA}}=0.25$, (c) $\delta_{\mathrm{NOAA}}=0.3$, (d) $\delta_{\mathrm{NOAA}}=0.35$ and (e) $\delta_{\mathrm{NOAA}}=0.4$ (2 September 1997). Marked areas in (b) and (c) will be compared in Fig. 15.

The SEIs in Fig. 10 are derived from Fig. 9(b) by applying the conditions in Eq. (5), where the threshold parameter $\delta_{\mathrm{NOAA}}$ is varied between 0.2 and 0.4 (Rectangular regions in Fig. 10(b) and (c) will be used in the discussion in Section 4). Comparison of Fig. 10(a)-(e) with Fig. 9(a) (difference image) indicates that the best agreement of the smoke region is attained for Fig. $10(\mathrm{c})$, which corresponds to $\delta_{\mathrm{NOAA}}=0.3$. When 
this figure is compared with Fig. 7(d) from the GMS VISSR, it is found that smoke regions are more separated in Fig. 10(c): this separation is particularly conspicuous in the southern part of the island. The number of thus separated regions is 89 for the entire region depicted in Fig. 10(c), whereas the number is only 28 in the case of VISSR. This fact suggests that higher spatial resolution of AVHRR results in the increase in the number of separated regions.

It turns out that when the spatial resolution of Fig. 10(c) is matched with that in Fig. 7(d), 96\% of pixels show agreement in the classification result between the two figures. Therefore, the present SEI results are satisfactorily consistent with each other. The threshold value of the $\delta$ parameter is 0.3 for both the VISSR and AVHRR cases. This coincidence is explained by the fact that the texture window size of $N_{x} \times$ $N_{y}=9 \times 9$ is sufficiently smaller than the smoke area for each case, regardless of the difference in the spatial resolutions. With regard to this window size effect, the following two cases are also examined. First, the spatial resolution of AVHRR is reduced by a factor of $1 / 4.5$, so that it becomes equal to the resolution of VISSR. When the identical window size of $9 \times 9$ (with the same threshold parameter of $\delta_{\mathrm{NOAA}}=0.3$ ) is adopted, we obtain a figure similar to Fig. 10(c). This result supports the interpretation of the equal values of $\delta$ mentioned above. Second, the AVHRR image is analyzed with an increase window size of $41 \times 41$. Although the area size corresponding to this enlarged window is equivalent to that of an VISSR window of $9 \times 9$ pixels, a figure similar to Fig. $10(\mathrm{c})$ is obtained when $\delta_{\mathrm{NOAA}}$ is increased to 0.5 . This increase is observed even for the "pure" smoke areas (i.e. no effect of background surfaces), and it is explained by the change in the distribution of elements of GLDV vectors.

Fig. 11 shows the variation in the number of smoke pixels on Kalimantan, plotted for 12 days in September

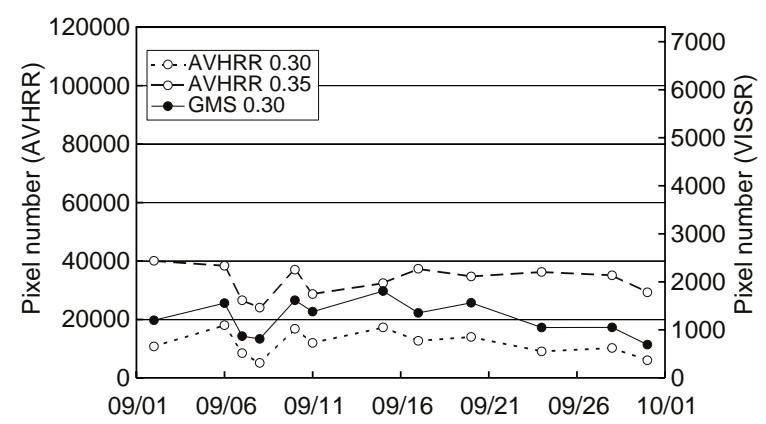

Fig. 11. Variation of the smoke pixel number (September 1997). Open circles show the smoke pixel number by the texture analysis from the AVHRR image: dashed line is with $\delta_{\mathrm{NOAA}}=0.35$ and dotted line with $\delta_{\mathrm{NOAA}}=0.3$. Closed circles are the smoke pixel number from the GMS image with $\delta_{\mathrm{GMS}}=$ 0.3 .
1997. Open circles depict the variation in the AVHRR SEI with $\delta_{\mathrm{NOAA}}=0.3$ (dotted line) and $\delta_{\mathrm{NOAA}}=0.35$ (broken line), while closed circles that in the VISSR SEI with $\delta_{\mathrm{GMS}}=0.3$ (solid line). In this figure, the left and right ordinates apply to the AVHRR and VISSR images, respectively. The relative scale of these two is given in accordance with the actual ratio (20.25:1) of the pixel numbers in the two cases. Thus, Fig. 11 suggests that in terms of the smoke pixel numbers, the VISSR result with $\delta_{\mathrm{GMS}}=0.3$ is consistent with the AVHRR result with $\delta_{\mathrm{NOAA}}=0.3-0.35$. We obtain a correlation coefficient of 0.91 between these VISSR and AVHRR $\left(\delta_{\mathrm{NOAA}}=0.3\right)$ results.

\subsection{Smoke optical thickness from AVHRR images}

Variation of the aerosol optical thickness at $550 \mathrm{~nm}, \tau_{550}$, is shown in Fig. 12 (open circles). This is obtained from the data on point A in Fig. 2 (above sea surface near Singapore) using the method described in Section 2.3. This figure also shows sky-radiometer data observed at Singapore (filled circles). A data point on November 10 exhibits a considerably large value of $\tau_{550}=1.3$, presumably due to local cloud above the observation site. Except for this point, the two data show a reasonable agreement with a correlation coefficient of 0.84 . Thus, it is concluded that the method in Section 2.3, particularly the assumption on the sea surface reflectivity $(2 \%)$, is appropriate in this case. The optical thickness is used in the following subsection to discuss the relationship between the texture threshold $\delta_{\mathrm{NOAA}}$ and the aerosol column amount.

\subsection{Relationship between the texture threshold value and aerosol column amount}

Here we discuss the relationship between the threshold value of the texture analysis $\delta_{\mathrm{NOAA}}$ and the

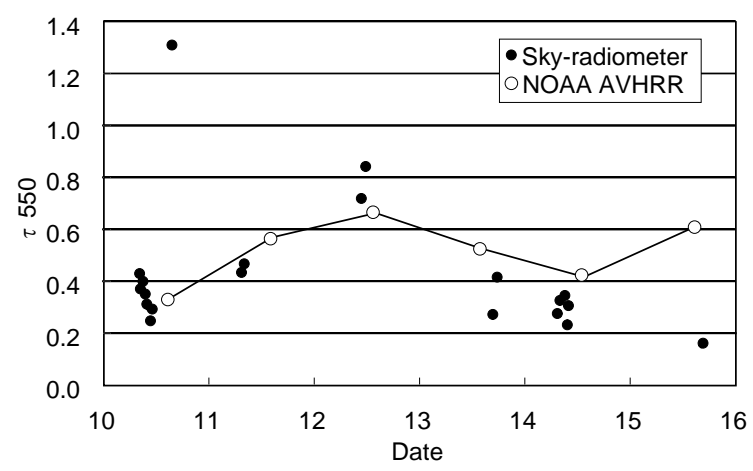

Fig. 12. Variation of $\tau_{550}$ at Singapore (November 1997). Open circles show $\tau_{550}$ observed by NOAA AVHRR ch. 1, and filled circles indicate $\tau_{550}$ observed by the sky-radiometer. 
coefficient of variation in the smoke optical thickness. Fig. 13 depicts the marked regions in Fig. 10(b) and (c) (the same rectangular area) in an expanded scale. Besides, the smoke regions in Fig. 10(c) (with $\left.\delta_{\text {NOAA }}=0.3\right)$ are illustrated in gray, while those in Fig. 10(b) (with $\delta_{\mathrm{NOAA}}=0.25$ ) in black. Regions designated as (a1)-(a4) are those classified as one smoke region when $\delta_{\mathrm{NOAA}}=0.3$, but separately classified in the case of $\delta_{\mathrm{NOAA}}=0.25$. The same applies to regions (b1)(b3), (c1)-(c2), and (d1)-(d2). Each of (a1)-(d2) is composed of $5 \times 5$ pixels. Table 2 lists the optical thickness $\tau_{550}$ obtained for each region using the method of Section 2.3. The parameter $\sigma$ in the table is the coefficient of variation defined as

$$
\sigma=\left[\frac{\sum_{i=1}^{N_{x}} \sum_{j=1}^{N_{y}}\left(\tau_{i j}-\overline{\tau_{550}}\right)^{2}}{\left(N_{x} N_{y}-1\right) \bar{\tau}_{550}^{2}}\right]^{1 / 2}
$$

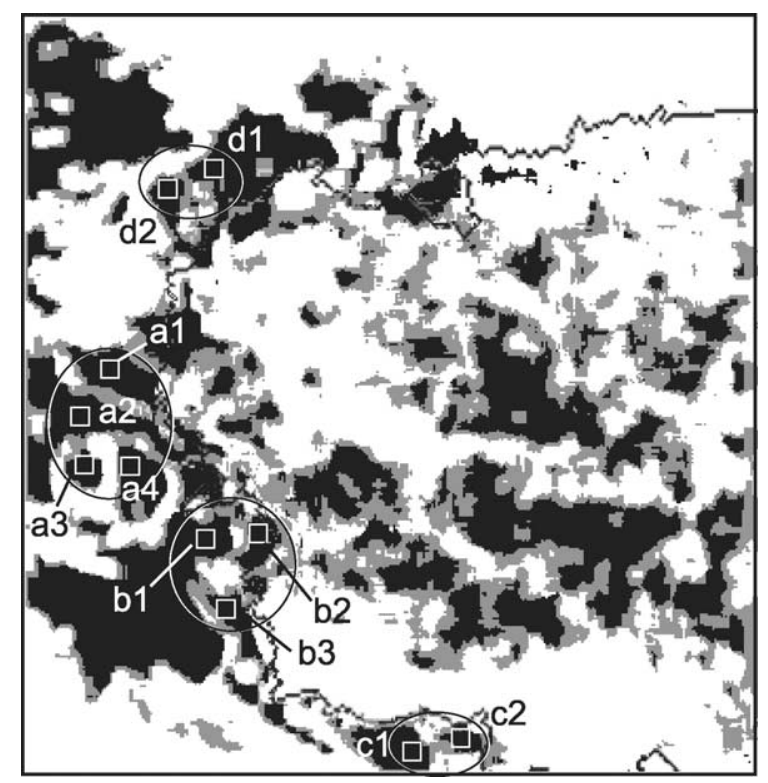

Fig. 13. Enlarged depiction of the marked areas in Fig. 10(b) and (c). (a1)-(a4), (b1)-(b3), (c1)-(c2) and (d1)-(d2) are the retrieval areas of $\tau_{550}(5 \times 5$ pixels $)$. where $\overline{\tau_{550}}$ denotes the average value of $\tau_{550}$ in the $5 \times 5$ window.

In Table 2, the average values are different among the four groups of (a1)-(a4), (b1)-(b3), (c1)-(c2), and (d1)-(d2). Thus, it is confirmed that the essential aspect of the smoke extraction by texture analysis is not to select regions with a particular value of $\tau_{550}$, but to detect regions with uniform values of $\tau_{550}$ in a satellite image. For this reason, the method becomes useful when combined with the spectral information such as those in Eqs. (3) and (5).

Next we consider the coefficient of variation $\sigma$ in each group. In group (a1)-(a4), the coefficient changes in a region of $0.06-0.08$ for $\delta_{\mathrm{NOAA}}=0.25$, and it increases to 0.09 when $\delta_{\mathrm{NOAA}}=0.3$. Similar tendency is also found for other three groups. Therefore, increase in the threshold value leads to increase in the coefficient of variation, as expected from the property of texture analysis.

\subsection{Inhomogenous smoke distribution}

So far we have dealt with cases where smoke detection is accomplished by considering the homogeneity in the texture window. The TAIs in Figs. 5(a) and 9(b), for instance, exhibit insignificant dependence on the angular parameter $\theta$, indicating little influence from wind transportation. When strong wind field is present, on the contrary, angular dependence could be brought about, as in cirrus studies by Kuo et al. (1988). Fig. 14 displays SEIs of the sea area surrounded by Kalimantan, Jawa, and Smatera islands on 8 October 1997, when the forest fire had subsided, but still emitted dense smoke. The wind direction was reported as indicated in Fig. 14(a), from east at Isakan (southern Kalimantan) and Tanjuang Panda (east Kalimantan), and from south at Jakarta (Ru et al., 2000). In (b)-(h), we show the results with $\theta=0^{\circ}, 15^{\circ}, \ldots, 90^{\circ}$. Evident striations are found along the North-South direction for $\theta=0^{\circ}$ and $90^{\circ}$ : they become inconspicuous as the angle is changed toward $\theta=45^{\circ}$, at which almost all the homogenous regions appear to connect with each other. This angular dependence seems to be consistent with the smoke

Table 2

Variance of $\tau_{550}$ in the areas (a1)-(d2) of Fig. 13

\begin{tabular}{|c|c|c|c|c|c|c|c|c|c|c|c|}
\hline Area & al & $\mathrm{a} 2$ & a3 & $\mathrm{a} 4$ & b1 & $\mathrm{b} 2$ & b3 & $\mathrm{cl}$ & c2 & $\mathrm{d} 1$ & $\mathrm{~d} 2$ \\
\hline$\overline{\tau_{550}}(\delta=0.25)$ & 2.61 & 2.35 & 2.44 & 2.57 & 1.08 & 1.49 & 1.33 & 1.36 & 1.63 & 1.78 & 2.06 \\
\hline$\overline{\tau_{550}}(\delta=0.30)$ & \multicolumn{4}{|c|}{2.49} & & 1.30 & & \multicolumn{2}{|c|}{1.50} & \multicolumn{2}{|c|}{1.93} \\
\hline$\sigma(\delta=0.25)$ & 0.07 & 0.08 & 0.06 & 0.07 & 0.05 & 0.09 & 0.09 & 0.07 & 0.03 & 0.05 & 0.02 \\
\hline$\sigma(\delta=0.30)$ & \multicolumn{4}{|c|}{0.09} & \multicolumn{3}{|c|}{0.15} & \multicolumn{2}{|c|}{0.10} & \multicolumn{2}{|c|}{0.08} \\
\hline
\end{tabular}


(a)

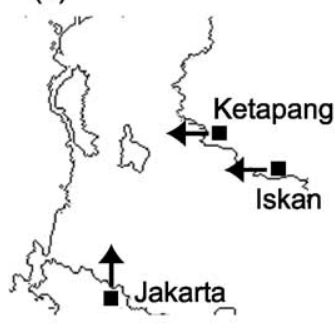

(d) $\theta=30^{\circ}$

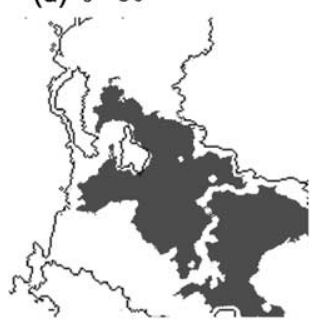

(g) $\theta=75^{\circ}$

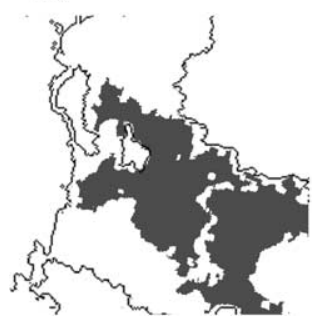

(b) $\theta=0^{\circ}$

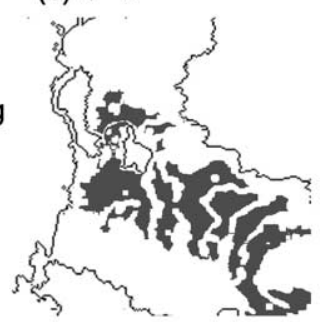

(e) $\theta=45^{\circ}$

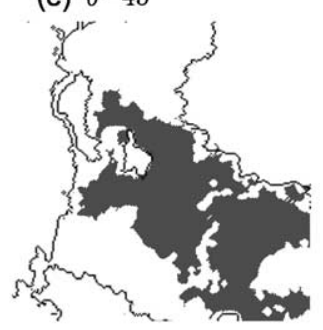

(h) $\theta=90^{\circ}$

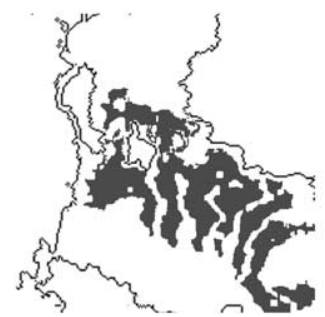

(c) $\theta=15^{\circ}$

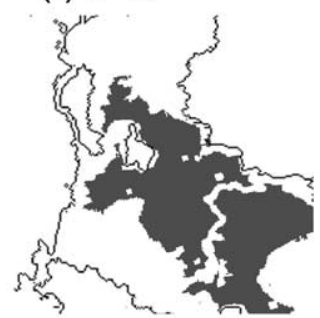

(f) $\theta=60^{\circ}$

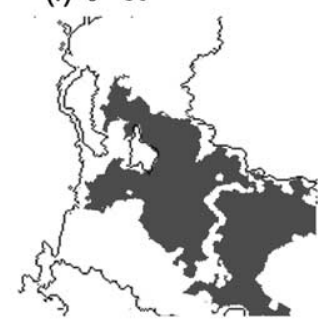

Fig. 14. Angular dependence of SEI (8 October 1997). (a) Wind direction surrounding Kalimantan, Java and Sumatra island. (b) SEI obtained with $\theta=0^{\circ}$, (c) $\theta=15^{\circ}$, (d) $\theta=30^{\circ}$, (e) $\theta=45^{\circ}$, (f) $\theta=60^{\circ}$, (g) $\theta=75^{\circ}$ and (h) $\theta=90^{\circ}$.

transportation expected from the wind direction data in Fig. 16(a), though more analysis using ancillary data would be required to elucidate the effect in detail. Nevertheless, this example demonstrates well that texture features can be exploited to study the smoke transportation above the ocean, where the background exhibits texture features sufficiently homogenous.

\section{Conclusions}

In this paper, we have analyzed satellite images of the Indonesian forest fire in 1997. Unsupervised, automatic extraction of fire smokes has been implemented by combining the multi-spectral and texture information. Comparison between GMS-5 VISSR and NOAA-14 AVHRR results has shown that when the threshold values of the texture analysis $\delta$ are properly chosen, good agreement can be obtained in spite of the marked difference in their spatial resolutions. We have used the textural mean obtained from GLDV representations to determine whether a region in an image is homogenous or not. This is basically the same as the analysis by using the coefficient of variation. In fact, the results of Section 3.4 confirms that the resulting homogeneity of the aerosol optical thickness in a texture window is determined by the threshold value, though it is also dependent on the window size employed. More detailed information about the smoke distribution is also available from the texture analysis, and we have briefly discussed the angular dependence of the textureanalyzed images. Such types of application would be particularly useful to detect transportation of fire smoke over the ocean.

\section{References}

Ackerman, S.A., Chang, H., 1992. Radiative effects of airborne dust on regional energy budgets at the top of the atmosphere. Journal of Applied Meteorology 31 (2), 223-233. 
Asakuma, K., Otsutsumi, S., Kubota, T., Yabuki, M., Kuze, H., Takeuchi, N., 2000. Retrieval of aerosol optical thickness from NOAA/AVHRR data and its application to the derivation over land area in Chiba. In: Proceedings of SPIE, 4150, Optical Remote Sensing of the Atmosphere and Clouds II, pp. 290-298.

Berendes, T.A., Kuo, K.S., Logar, A.M., Corwin, E.M., Welch, R.M., Baum, B.A., Pretre, A., Weger, R.C., 1999. A comparison of paired histogram, maximum likelihood, class elimination, and neural network approaches for daylight global cloud classification using AVHRR imagery. Journal of Geophysical Research 104 (D6), 6199-6213.

Charlson, R.J., Schwartz, S.E., Hales, J.M., Cess, R.D., 1992. Climate forcing by anthropogenic aerosols. Science 255, 423-430.

Christopher, S.A., Kliche, D.V., Joyce, C., Welch, R.M., 1996. First estimates of the radiative forcing of aerosols generated from biomass burning using satellite data. Journal of Geophysical Research 101 (D16), 21265-21273.

Ebert, E., 1987. A pattern recognition technique for distinguishing surface and cloud types in the polar regions. Journal of Climate and Applied Meteorology 26, 14131427.

Holben, B.N., Setzer, A., Eck, T.F., Pereira, A., Slutsker, I., 1996. Effect of dry season biomass burning on Amazon basin aerosol concentrations and optical properties, 19921994. Journal of Geophysical Research 101 (D14), 1946519481.

Kaufman, Y.J., Tucker, C.J., Fung, I., 1990. Remote sensing of biomass burning in tropics. Journal of Geophysical Research 95, 9927-9939.

Kaufman, Y.J., Holben, B.N., 1996. Hemispherical backscattering by biomass burning and sulfate particles derived from sky measurements. Journal of Geophysical Research 101 (D14), 19433-19445.

Key, J., 1990. Cloud cover analysis with Arctic Advanced Very High Resolution Radiometer data, 2. Classification with spectral and textural measures. Journal of Geophysical Research 95 (D6), 7661-7675.

Kuo, K.S., Welch, R.M., Sengupta, S.K., 1988. Structural and textural characteristics of cirrus clouds observed using high spatial resolution LANDSAT imagery. Journal of Applied Meteorology 27, 1242-1260.

Liousee, C., Dulac, F., Cachier, H., 1997. Remote sensing of carbonaceous aerosol production by African savanna biomass burning. Journal of Geophysical Research 102 (D5), 5893-5911.

Lubin, D., Morrow, E., 1998. Evaluation of AVHRR cloud detection and classification method over the central Arctic ocean. Journal of Applied Meteorology 37, 166-183.

Nakajima, T., Higurashi, A., 1997. AVHRR remote sensing of aerosol optical properties in the Persian gulf region. Journal of Geophysical Research 102 (D14), 16935-16946.

Nair, U.S., Weger, R.C., Kuo, K.S., Welch, R.M., 1998. Clustering, randomness, and regularity in cloud fields 5 . The nature of regular cumulus cloud fields. Journal of Geophysical Research 103, 11363-11380.

Prins, E.M., Menzel, W.P., 1994. Trends in south American biomass burning detected with GOES visible infrared spin scan radiometer atmospheric sounder from 1983 to 1991. Journal of Geophysical Research 99 (D8), 16719-16753.

Rao, C.R.N., Stowe, L.L., McClain, E.P., 1997. Remote sensing of aerosol over oceans using AVHRR data: theory, practice and application. International Journal of Remote Sensing 10, 743-749.

Rao, C.R.N., Chen, J., 1996. Revised post-launch calibration of channels 1 and 2 of the Advanced Very High Resolution Radiometer on board the NOAA-14 spacecraft. NOAASIS Home Page, (psbsgil.nesdis.noaa.gov:8080/EBB $/ \mathrm{ml} /$ niccall.html) \& (psbsgil.nesdis.noaa.gov:8080/EBB $/ \mathrm{ml} /$ niccal2.html).

Ru, J., Kaneta, S., Minomura, M., Kuze, H., Takeuchi, N., 2000. Study on the biomass burning aerosol in Indonesian forest fire by using AVHRR two channel data. Remote Sensing Society of Japan 20, 19-30.

Taylor, K.E., Penner, J.E., 1994. Climate system response to aerosols and greenhouse gases: a model study. Nature 369, 734-737.

Vermote, E.F., Tanre, D., Deuze, J.L., Herman, M., Morcrette, J.J., 1997a. Second simulation of the satellite signal in the solar spectrum, 6S: an overview. IEEE Transactions on Geoscience and Remotesensing 35 (3), 675-686.

Vermote, E.F., Tanre, D., Deuze, J.L., Herman, M., Morcrette, J.J., 1997b. 6S User Guide Version 2.

Weszka, J.S., Charles, R.D., Rosenfeld, A., 1976. A comparative study of texture measures for terrain classification. IEEE Transactions on Systems Man and Cyberneties SMC6, 269-285. 